\title{
CORRECTION
}

\section{Correction to: TAOK1 negatively regulates IL-17-mediated signaling and inflammation}

\author{
Zhaoru Zhang ${ }^{1}$, Zhen Tang ${ }^{2}$, Xianwei Ma ${ }^{3}$, Kai Sun ${ }^{4}$, Liping Fan ${ }^{4}$, Jie Fang ${ }^{5}$, Jianping Pan ${ }^{5}$, Xiaojian Wang ${ }^{6}$, Huazhang An ${ }^{2}$ and
} Jun Zhou ${ }^{1}$

Cellular \& Molecular Immunology (2018) 15:940; https://doi.org/10.1038/s41423-018-0048-5

Correction to: Cellular \& Molecular Immunology (2018). https://doi. org/10.1038/cmi.2017.158; published online 5 February 2018

In this article, published online 5 February 2018, the second primer of mouse actin, il-6, il-1 $\beta$, TNFa, cxcl1, cxcl2 and $\mathrm{ccl} 20$ in
Table 2 should be marked as "reverse primer" instead of "forward primer". Corrected Table 2 is shown below. The authors regret the errors.

\begin{tabular}{|c|c|}
\hline human gapdh reverse primer & 5'-GGATCTCGCTCCTGCAAGATG-3' \\
\hline human il-6 reverse primer & 5'-GGGAAGGCAGCAGGCAACAC-3' \\
\hline human $\mathrm{cxcl} 1$ forward primer & 5'-AGTCATAGCCACACTCAAGAATGG-3' \\
\hline human $\mathrm{cxcl} 1$ reverse primer & 5'- GATGCAGGATTGAGGCAAGC-3' \\
\hline human $\mathrm{cxc} 22$ reverse primer & 5'-AAACACATTAGGCGCAATCC-3' \\
\hline human $\mathrm{ccl} 20$ forward primer & 5'-GCGCAAATCCAAAACAGACT-3' \\
\hline human $\mathrm{ccl} 20$ reverse primer & 5'-CAAGTCCAGTGAGGCACAAA-3' \\
\hline mouse actin forward primer & 5'- AGTGTGACGTTGACATCCGT-3' \\
\hline mouse actin reverse primer & 5'-GCAGCTCAGTAACAGTCCGC-3' \\
\hline mouse TNF $\alpha$ forward primer & 5'-AAGCCTGTAGCC CACGTCGTA-3' \\
\hline mouse $\mathrm{TNF} \alpha$ reverse primer & 5'-GGCACCACTAGTTGGTTGTCTTTG-3' \\
\hline mouse $\mathrm{cxcl} 1$ forward primer & 5'-CGCTTCTCTGTGCAGCGCTGCTGCT-3' \\
\hline mouse $\mathrm{cxcl} 1$ reverse primer & 5'-AAGCCTCGCGACCATTCTTGAGTC- $3^{\prime}$ \\
\hline mouse $\mathrm{cxcl} 2$ forward primer & 5'-CCTGGTTCAGAAAATCATCCA-3' \\
\hline mouse $\mathrm{cxcl} 2$ reverse primer & 5'-CTTCCGTTGAGGGACAGC-3' \\
\hline mouse $\mathrm{ccl} 20$ forward primer & 5'-AACTGGGTGAAAAGGGCTGT-3' \\
\hline mouse $\mathrm{ccl} 20$ reverse primer & 5'-GTCCAATTCCATCCCAAAAA-3' \\
\hline
\end{tabular}

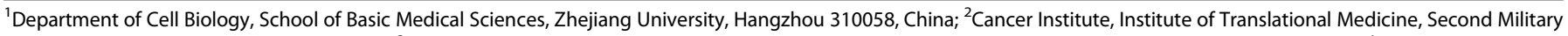

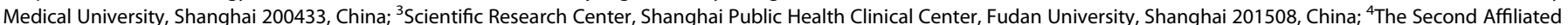

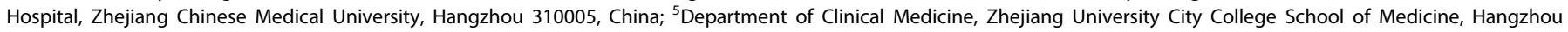
310015, China and ${ }^{6}$ Institute of Immunology, School of Medicine, Zhejiang University, Hangzhou 310058, China

Correspondence: Jun Zhou (zhjjwm300@zju.edu.cn)

These authors contributed equally: Zhaoru Zhang, Zhen Tang.
}

Published online: 29 May 2018 